\title{
Applications to Mathematical Finance
}

\author{
Freddy Delbaen, Eidgenössische Technische Hochschule, Zürich \\ Walter Schachermayer, Technische Universität, Wien
}

December 19, 2001

\begin{abstract}
We give an introduction to the theory of Mathematical Finance with special emphasis on the applications of Banach space theory.

The introductary section presents on an informal and intuitive level some of the basic ideas of Mathematical Finance, in particular the notions of "No Arbitrage" and "equivalent martingale measures". In section two we formalize these ideas in a mathematically rigorous way and then develop in the subsequent four sections some of the basic themes.

Of course, in this short handbook-contribution we are not able to give a comprehensive overview of the whole field of Mathematical Finance; we only concentrate on those issues where Banach space theory plays an important role.
\end{abstract}




\section{Introduction}

The field of Mathematical Finance has undergone a remarkable development since the seminal papers by F. Black and M. Scholes [BS73] and R. Merton [M73], in which the famous "Black-Scholes Option Pricing Formula" was derived. In 1997 the Nobel prize in Economics was awarded to R. Merton and M. Scholes for this achievement, thus also honoring the late F. Black.

The idea of developing a "formula" for the price of an option actually goes back as far as 1900, when L. Bachelier [B00] wrote a thesis under the supervision of H. Poincaré with the title "Théorie de la spéculation". His aim was precisely to obtain such a formula to be used on the stock market in Paris, which was also booming at the previous fin de siècle.

Bachelier's contribution was remarkable in several respects: firstly he had the innovative idea of using a stochastic process as a model for the price evolution of a stock. He had an almost mystic belief in the "law of probability":

Si, á l'égard de plusieurs questions traitées dans cette étude, j'ai comparé les résultats de l'observation à ceux de la théorie, ce n'était pas pour vérifier des formules établies par les méthodes mathématiques, mais pour montrer seulement que le marché, à son insu, obéit à une loi qui le domine: la loi de la probabilité.

For a stochastic process $\left(S_{t}\right)_{0 \leq t \leq T}$ he made a natural and far-reaching choice being the first to give a mathematical definition of Brownian motion: he required that, for $0 \leq t \leq u \leq T$, the random variable $S_{u}-S_{t}$ is normally distributed with mean zero and variance $\sigma^{2}(u-t)$, where $\sigma^{2}>0$ is a fixed parameter, and, that for $0 \leq t_{0} \leq \cdots \leq t_{n} \leq T$ the random variables $\left(S_{t_{i}}-S_{t_{i-1}}\right)_{i=1}^{n}$ are independent. If we also fix the real number $S_{0}$ we have well defined a stochastic process, namely Brownian motion, which in the present context is interpreted as follows: $S_{0}$ is today's (known) price of a stock (say a share of company XYZ to fix ideas) while for the time $t>0$ the price $S_{t}$ is a normally distributed random variable.

Bachelier's use of Brownian motion in the context of finance was thus prior to the use of this process in the context of physics by A. Einstein [E05] and, independently, by M. Smoluchowski [Sm16] some five years later.

Having fixed the model, Bachelier now turned to the question of pricing an option (to be precise: a European call option); such an option is the right to buy one unit of the underlying stock at a fixed time $T$ (the expiration time) and at a fixed price $K$ (the strike price). This determines the value $C_{T}$ of the option at time $T$ as a function of the (unknown) value $S_{T}$ of the stock at time $T$, namely

$$
C_{T}=\left(S_{T}-K\right)_{+} \cdot
$$

Indeed, a moment's reflection reveals that the option is worthless (at time $T)$ if $S_{T} \leq K$, and is worth the difference between $S_{T}$ and $K$ if $S_{T}>K$ : in 
the latter case the holder of the option will exercise the option to buy one unit of stock at a price $K$ and can immediately resell it at the present market price $S_{T}$ to make a profit $S_{T}-K$. (In practice, a cash settlement is often made.)

Hence we know, conditionally on the random variable $S_{T}$, what the option will be worth at time $t=T$. But what we really want to know is what the option is worth today, i.e., at time $t=0$. To determine this quantity Bachelier simply took the expected value, i.e.,

$$
C_{0}:=\mathbb{E}\left[C_{T}\right]=\mathbb{E}\left[\left(S_{T}-K\right)_{+}\right]
$$

which leads to a simple explicit formula for $C_{0}$ involving the normal distribution function. The approach of formula (2) has been used in actuarial mathematics for centuries and is based on a belief in the law of large numbers: if the stochastic model $\left(S_{t}\right)_{0 \leq t \leq T}$ describes reality properly and an economic agent "frequently" buys an option at the price given in (2) then in average she will neither gain nor lose money.

After this brief sketch of Bachelier's work at the previous turn of the century several questions must be addressed, e.g.:

1. Is Brownian motion a "good model" for the price evolution of stocks?

2. Is the argument based on the law of large numbers really an economically sound basis for applying (2)?

Apart from these two basic questions there are plenty of other ones, e.g., the (minor) question of introducing some interest rate - or some drift of the process $S$ - into the model (which Bachelier safely ignored as he only was interested in short run options and also at the time interest rate was very low).

Bachelier's work was not appreciated by the contemporary economic literature and also in the mathematical literature it did not have the impact it should have deserved (it was, however, not forgotten by mathematicians: for example, it is quoted in Kolmogoroff's book [K33]).

Only in 1965 did the renowned economist and Nobel prize winner, P.R. Samuelson [S65] take up again the theme of designing an appropriate model for a stock price process: He proposed geometric Brownian motion with parameters $\mu \in \mathbb{R}$ and $\sigma^{2}>0$ obeying the stochastic differential equation

$$
d S_{t}=\mu S_{t} d t+\sigma S_{t} d B_{t}
$$

where $\left(B_{t}\right)_{0 \leq t \leq T}$ is a standard Brownian motion starting at $B_{0}=0$. Using Itô's formula one quickly verifies that the solution to (3) is given by the process

$$
S_{t}=S_{0} \exp \left(\left(\mu-\frac{\sigma^{2}}{2}\right) t+\sigma B_{t}\right)
$$

which is called geometric Brownian motion. If returns are defined through the logarithm, then the price process $S_{t}$ gives returns that are stationary and 
are independent when taken over disjoint time periods. It is a well known result that the only Lévy processes with this property and continuous paths are Brownian motions with drift. This immediately leads to (3) or (4).

The rationale of (3) is that the drift as well as the noise term driving the process $S_{t}$ is proportional to $S_{t}$; in other words, the difference between Brownian motion with drift, i.e., $S_{t}=S_{0}+a t+b B_{t}$ and geometric Brownian motion with drift as defined in (4) is similar to the difference between the methodology of calculating linear or compound interest. Clearly, in the short run there is little difference between the linear and the exponential point of view (in the deterministic as well as in the stochastic case) while, in the long run, the difference is very noticeable; it also is obvious, that the exponential point of view is economically more meaningful.

The model (4) of geometric Brownian motion today became the standard reference model to describe the price evolution of a stock; although promoted by Samuelson, it now is often called the Black-Scholes model or even the BlackScholes world.

It has many very appealing properties but its match to reality is not very good in many aspects (as is very well-known to practitioners): there are several key properties observed in real financial data (e.g., heavy tails, volatility clustering etc.) which are not captured by this model. Many alternative models have been proposed (e.g., in the early work of B. Mandelbrot [M66]). Hence, turning back to question 1. raised above - whether Brownian motion is the good model - it is generally agreed that geometric Brownian motion with drift is economically more reasonable than Bachelier's original choice (note, however, that in the short run there is little difference), but the question whether (geometric) Brownian motion is a "good model", cannot be answered with a simple yes or no: it depends on the context and purpose of the modeling.

We now turn to question 2 pertaining to the economic soundness of the pricing argument based on the law of large numbers: it was the merit of Black, Scholes [BS73] and Merton [M73] to have replaced this argument by a "no arbitrage" argument, which is of central importance to the entire theory. The basic principle underlying this idea is nicely explained in the subsequent little story quoted from a paper of B. Dupire [D93]:

Imagine you are offered a strange bet. A coin is tossed; you win $\$ 60$ if it comes up heads and lose $\$ 40$ for tails. Would you accept the bet?

It looks attractive: the expectation is $(0.50 \times 60)+(0.50 \times-40)=10$, a healthy bias. A more thorough examination may lead to second thoughts, however. Perhaps the pain of losing $\$ 40$ would overweight the pleasure of making $\$ 60$. What if it were $\$ 60,000$ and $\$ 40.000$ ? What if the coin were unbalanced, or tossed by a manipulator who can get tails three times out of four? 
In other words, your decision would depend on both preferences (risk aversion) and expectations, and it may well be wiser to refuse this apparently attractive proposition.

Let us now set the stage for a very similar situation. You are in a casino, at a simplified roulette table. There are only two possible outcomes: red or black. There is no zero and it is possible to play with the casino on a 1:1 basis. The proposed bet is: win $\$ 60$ if red comes up and lose $\$ 40$ for black. Would you accept?

At first sight, this seems equivalent to the previous bet, so you would decline the invitation. But the fact that it is possible to play with the casino makes a real difference. You can hedge the bet and build up a certain gain, regardless of the outcome - an arbitrage, in other words. Just accept the bet and, at the same time, put a stake of $\$ 50$ on black with the casino.

If red does come up, you make $\$ 60$ through the bet and lose $\$ 50$ with the casino. If black wins, you lose $\$ 40$ through the bet and make $\$ 50$ with the casino.

In either case, you are better off by \$10: the expected, potential gain has been converted into a certain one. Most important, this is true regardless of the real probabilities of red versus black, which could very well be 10:90.

Summing up: an arbitrage - which is the concept crystallized in the second part of the above story - is a riskless way of making a profit with zero net investment. This intuitive notion will be mathematically formalized in section 2 below.

An economically very reasonable assumption on a financial market consists of requiring that there are no arbitrage opportunities. (To convince yourself that this assumption is indeed reasonable, just think for a moment, what would happen in a liquid financial market offering arbitrage opportunities.) The remarkable fact is that this simple and primitive "principle of no arbitrage" allows already to determine a unique option price in the Black-Scholes model: to be slightly more formal, we assume that in our financial market there are two traded assets, the stock $S=\left(S_{t}\right)_{0 \leq t \leq T}$ whose price process is given by (4) above and a riskless bond $B$ whose price process $\left(B_{t}\right)_{0 \leq t \leq T}$ evolves deterministically with a constant interest rate $r$, i.e., $B_{t}=B_{0} e^{r t}$. For (mainly notational) simplicity we assume $r=0$.

We denote by $\left(\mathcal{F}_{t}\right)_{0 \leq t \leq T}$ the (right continuous, saturated) filtration generated by the process $S$ (which is defined on some stochastic base $(\Omega, \mathcal{F}, \mathbb{P})$ ). We then have that every $\mathcal{F}_{T}$-measurable random variable $f$ (satisfying an appro- 
priate integrability condition) can uniquely be written as

$$
\begin{aligned}
f & =c+\int_{0}^{T} H_{u} d S_{u} \\
& =c+(H \cdot S)_{T}
\end{aligned}
$$

for some constant $\mathrm{c}$ and a predictable process $\left(H_{t}\right)_{0 \leq t \leq T}$ (also satisfying an appropriate regularity condition). The interpretation of (5) applied to the $\mathcal{F}_{T}$-measurable random variable $f$ goes as follows: $f$ represents a "derivative security" which pays $f(\omega)$ at time $T$ if the "state of the world" $\omega \in \Omega$ is revealed at time $T$; a typical example is the European call option $f(\omega)=\left(S_{T}(\omega)-K\right)_{+}$ encountered above. Formula (5) states that you can replicate the derivative $f$ at an initial cost given by the constant $c$ and subsequently trading in the stock $S$ as prescribed by the predictable trading strategy $H$. Equation (5) combined with the principle of no arbitrage therefore implies that the only reasonable price of the derivative security $f$ must be equal to $c$. Indeed, if the present market price of the derivative security $f$ differs from the constant $c$ above one can make an arbitrage very similarly to the above casino example. We have thus derived - under the assumptions of the Black-Scholes model - a unique price for options by only using the no-arbitrage principle.

What is behind the integral representation formula (5)? To understand this formula assume first that $\mu=0$ in (4) above so that the process $S$ is a martingale. In this case formula (5) follows from the martingale representation theorem for the Brownian filtration (see, e.g. [RY91]).

In its simplest form this theorem states that any function $f \in L^{2}\left(\Omega, \mathcal{F}_{T}, \mathbb{P}\right)$ with $E[f]=0$, where $\left(\mathcal{F}_{t}\right)_{0 \leq t \leq T}$ is the filtration generated by a standard Brownian motion $\left(B_{t}\right)_{0 \leq t \leq T}$, can uniquely be written as a stochastic integral $f=\int_{0}^{T} \widetilde{H}_{u} d B_{u}$ where $\widetilde{H}$ is a predictable process such that the process $\left((\widetilde{H} \cdot B)_{t}\right)_{0 \leq t \leq T}=\left(\int_{0}^{t} \widetilde{H}_{u} d B_{u}\right)_{0 \leq t \leq T}$ is an $L^{2}$-bounded martingale. It is trivial, using (3) and letting $H_{t}=\frac{\widetilde{H}_{t}}{\sigma S_{t}}$, to rewrite this as an integral on $S$ (remember that $\mu=0)$ :

$$
f=\int_{0}^{T} \widetilde{H}_{u} d B_{u}=\int_{0}^{T} \widetilde{H}_{u} \frac{d S_{u}}{\sigma S_{u}}=\int_{0}^{T} H_{u} d S_{u} .
$$

Hence, admitting the above cited martingale representation theorem, we get the representation (5) for an arbitrary $f \in L^{2}(\Omega, \mathcal{F}, \mathbb{P})$ where $c=\mathbb{E}[f]$.

Hence we essentially find the same methodology as used by Bachelier and by actuaries: calculate prices by taking expectations. The reasoning behind this procedure however is now completely different; the argument involving the strong law of large numbers has been replaced by the (economically much more compelling) no arbitrage argument.

What happens in the case $\mu \neq 0$ ? Here the crucial trick is to pass from the original measure $\mathbb{P}$ to an equivalent measure $\mathbb{Q}$ such that under $\mathbb{Q}$ the process $S$ becomes a martingale. The determination of the Radon-Nikodym 
density of the measure $\mathbb{Q}$ with respect to the original measure $\mathbb{P}$ is the theme of the Cameron-Martin-Girsanov theorem (see, e.g. [RY91]): under the above assumptions the measure $\mathbb{Q} \ll \mathbb{P}$ under which $S$ is a martingale is unique, and its density is given by

$$
\frac{d \mathbb{Q}}{d \mathbb{P}}=\exp \left(-\frac{\mu}{\sigma} B_{T}-\frac{\mu^{2}}{\sigma^{2}} T\right)
$$

Note that $\frac{d \mathbb{Q}}{d \mathbb{P}}>0 \mathbb{P}$-a.s. so that the measures $\mathbb{Q}$ and $\mathbb{P}$ are equivalent, i.e., have the same nullset.

Now apply the above reasoning to the process $S$ defined on $\left(\Omega, \mathcal{F},\left(\mathcal{F}_{t}\right)_{0 \leq t \leq T}, \mathbb{Q}\right) ;$ to find, for $f \in L^{2}\left(\Omega, \mathcal{F}_{T}, \mathbb{Q}\right)$, again the representation $(5)$ for a suitable trading strategy $H$. We now have

$$
c=E_{\mathbb{Q}}[f]
$$

where $E_{\mathbb{Q}}[$.$] denotes expectation taken with respect to \mathbb{Q}$. Using again the principle of no arbitrage we find that $c$ is the only possible price for $f$ not allowing arbitrage opportunities (in the mathematical formalization of the no arbitrage principle given below we shall see that this principle remains unchanged under an equivalent change of measure; the only thing that matters are the sets of measure zero).

For a European call option $f=\left(S_{T}-K\right)_{+}$the constant $c$ in (8) can be explicitly calculated yielding the celebrated Black-Scholes option pricing formula.

Summing up the above methodology: we have passed from the original measure $\mathbb{P}$ to a so-called risk-neutral measure $\mathbb{Q}$, which in the Black-Scholes model turned out to be unique, and then have used this measure to calculate the price of a derivative $f$ via (8); we have claimed that this is the unique arbitrage-free price for $f$.

The above presentation was deliberately informal and should only motivate the questions which are dealt with in full mathematical rigor in the subsequent sections. For example: What happens for more general stochastic processes $\left(S_{t}\right)_{0 \leq t \leq T}$ ? What is the exact relation between the passage to the risk-neutral measure and the no arbitrage considerations?

The latter question is the theme of the so-called Fundamental Theorem of Asset Pricing which-roughly speaking - states that a process $S=\left(S_{t}\right)_{0 \leq t \leq T}$ does not allow arbitrage opportunities if and only if there is an equivalent measure $\mathbb{Q}$ under which $S$ is a martingale.

The history of the fundamental theorem of asset pricing goes back to the seminal papers of Harrison, Kreps and Pliska written in the late seventies ([HK79], [HP81], [K81]): In these papers the methodology originally developed in a somewhat ad hoc way for the Black-Scholes model was put in a general framework. It turned out that the theory of stochastic integration, as 
developed notably in Strasbourg in the sixties and seventies (see, e.g., [DM80]), was taylor-made for the treatment of stochastic processes arising in finance.

After the pioneering work of Harrison, Kreps and Pliska many authors made contributions to gradually improve the understanding about this fundamental theorem, e.g., Duffie-Huang [DH86], Stricker [S90], Dalang-Morton-Willinger [DMW90] etc.

It turns out that, for general stock price processes $S$, it is quite hard to state and prove sharp and mathematically sound versions of the fundamental theorem and that a good amount of Banach space theory is needed for the proof and for its motivation. This program was accomplished in [DS94] and [DS98].

We shall deal with this issue in section 3 below after having formalized the notions of arbitrage and trading strategies in section 2. In section 4 we investigate the case of continuous processes $\left(S_{t}\right)_{0 \leq t \leq T}$ which allows for some sharper results than the general case dealt with in sections 2 and 3.

In section 5 we analyze how the no-arbitrage-considerations are effected by changes of numéraire (e.g., measuring the value of assets in Euros or in US \$) which naturally leads to the definition of a Banach space of stochastic integrals. Finally in section 6 we analyze the question of finding necessary and sufficient conditions for the closedness of an appropriately defined space of stochastic integrals in $L^{2}(\Omega, \mathcal{F}, \mathbb{P})$; this closedness is obviously crucial if one wants to use orthogonal projections in $L^{2}$. It turns out that these questions are intimately related to martingale theory in $H^{1}$ and $\mathrm{BMO}$, weighted norm inequalities and reverse Hölder inequalities.

We have selected these topics of the field of Mathematical Finance because of their intimate relationship with Banach space theory. Although the Fundamental Theorem of Asset Pricing crucial for the foundation of the theory as the name rightly indicates - it only is the door-opener to a wide range of questions. For a more comprehensive presentation of this fast growing field we refer to the recent text-book literature (e.g. [LL96], [MR97], [B98], [KS98], [S99] and the references given there).

\section{Strategies and Arbitrage Possibilities}

As stated in the introduction the main ingredient in arbitrage theory is stochastic integration. In this section we will give a mathematical formulation of the objects referred to in the introduction. All processes are defined on a stochastic basis $\left(\Omega,\left(\mathcal{F}_{t}\right)_{t>0}, \mathbb{P}\right)$ where $\mathcal{F}_{t}$ describes an increasing family of $\sigma$-algebras on $\Omega$. The probability measure $\mathbb{P}$ is defined on the $\sigma$-algebra $\mathcal{F}_{\infty}=\bigvee_{t \geq 0} \mathcal{F}_{t}$. The $\sigma$-algebra $\mathcal{F}_{t}$ can be seen as the mathematical description of the information available at time $t$. As common in stochastics, we will also assume that the filtration $\mathcal{F}$ satisfies the usual hypotheses, i.e., the filtration is right continuous, 
meaning that $\mathcal{F}_{t}=\cap_{s>t} \mathcal{F}_{s}$, and the $\sigma$-algebra $\mathcal{F}_{0}$ contains all the null sets of $\mathcal{F}_{\infty}$. To avoid trivialities at time $t=0$ we assume that $\mathcal{F}_{0}$ only consists of the null sets and their complements. By taking $\mathbb{R}_{+}$as the time set we aim for the most general situation. It is obvious that models with finite time horizon can be imbedded in a model where the time set is $\mathbb{R}_{+}$.

The time an agent wants to buy or sell a financial instrument can be a random variable, but it may only depend on past information and hence it must be a stopping time.

Definition 2.1 A stopping time $T$ is a random variable $T: \Omega \rightarrow \mathbb{R}_{+} \cup\{+\infty\}$ such that for each $t \geq 0$, we have that $\{T \leq t\} \in \mathcal{F}_{t}$. If $T_{1} \leq T_{2}$ are two stopping times then the stochastic interval $\llbracket T_{1}, T_{2} \rrbracket$ is the set

$$
\llbracket T_{1}, T_{2} \rrbracket=\left\{(t, \omega) \mid t \in \mathbb{R}_{+} ; T_{1}(\omega) \leq t \leq T_{2}(\omega)\right\} .
$$

Other intervals $\rrbracket T_{1}, T_{2} \rrbracket, \llbracket T_{1}, T_{2} \llbracket, \rrbracket T_{1}, T_{2} \llbracket$ are defined in an analogous way. The smallest $\sigma$-algebra on $\mathbb{R}_{+} \times \Omega$ containing the stochastic intervals of the form $\rrbracket T_{1}, T_{2} \rrbracket$ ( $T_{1}$ and $T_{2}$ are stopping times) is called the predictable $\sigma$-algebra $\mathcal{P}$. A process $H: \mathbb{R}_{+} \times \Omega \rightarrow \mathbb{R}^{d}$ is called predictable if it is measurable with respect to the predictable $\sigma$-algebra.

Mathematically a process $S$, modeling the price evolution of $d$ stocks, is best described by a $d$-dimensional semi-martingale $S: \mathbb{R}_{+} \times \Omega \rightarrow \mathbb{R}^{d}$. The set $\mathbb{R}_{+}$is the set of times where trading can take place. As in the introduction we also suppose that there is a riskless bond $\left(B_{t}\right)_{0 \leq t}$ traded in the market. By expressing the prices of the stocks in terms of the bond (see section 4 below) we may assume without loss of generality that $B_{t} \equiv 1$, for all $t$.

We will see, that in the case where $S$ is locally bounded, the semi-martingale condition is needed to obtain a sound model and therefore does not restrict the generality of the setting.

We recall from the general theory (e.g., [DM80]) that a process $S=\left(S_{t}\right)_{t \geq 0}$ is locally bounded if there is a sequence of stopping times $\left(T_{n}\right)_{n \geq 1}$ such that $T_{n} \leq T_{n+1}, \lim _{n \rightarrow \infty} T_{n}=+\infty$ almost surely, and $S$ is bounded on the intervals $\llbracket 0, T_{n} \rrbracket$, i.e., there are $k_{n} \in \mathbb{R}$ such that for all $t, 0 \leq t \leq T_{n}(\omega)$ : $\left|S_{t}(\omega)\right| \leq k_{n}$.

A stochastic process $S$ which is càdlàg (i.e., right continuous with left limits) is a semi-martingale if we can build a stochastic integration theory with it. To give a precise definition requires some extra notations and definitions.

Definition 2.2 A process $H: \mathbb{R}_{+} \times \Omega \rightarrow \mathbb{R}^{d}$ is called simple predictable if there is a finite sequence of stopping times $0=T_{0} \leq T_{1} \leq \ldots \leq T_{n+1}<\infty$, $\mathbb{R}^{d}$-valued functions $f_{0}, \ldots f_{n}$ such that each $f_{j}$ is $\overline{\mathcal{F}}_{T_{j}}$-measurable and $H=$ $\sum_{j=1}^{n} f_{j} \mathbf{1}_{\llbracket T_{j}, T_{j+1} \rrbracket}$.

It is easily seen that such a simple process is indeed measurable for the predictable $\sigma$-algebra. Let us denote by $\mathfrak{S}_{\infty}$ the space of all bounded simple predictable process. On $\mathfrak{S}_{\infty}$ we define the norm $\|H\|_{\infty}=\sup _{t, \omega}\left|H_{t}(\omega)\right|$. 
If $S$ is a $d$-dimensional càdlàg process then we can define the stochastic integral in an elementary way:

$$
\begin{aligned}
(H \cdot S)_{\infty} & =\int_{[0, \infty]} H_{u} d S_{u} \\
& =\sum_{i=1}^{d} \sum_{j=1}^{n} f_{j}^{i}\left(S_{T_{j+1}}^{i}-S_{T_{j}}^{i}\right)
\end{aligned}
$$

The indefinite integral defines a càdlàg process.

$$
\begin{aligned}
(H \cdot S)_{t} & =\int_{[0, t]} H_{u} d S_{u} \\
& =\sum_{i=1}^{d} \sum_{j=1}^{n} f_{j}^{i}\left(S_{T_{j+1} \wedge t}^{i}-S_{T_{j} \wedge t}^{i}\right)
\end{aligned}
$$

The notion of stochastic integral has an immediate financial interpretation. If at times $\left(T_{j}\right)_{0 \leq j \leq n}$ the trader decides to hold the position $\left(f_{j}\right)$ during the time period $\rrbracket T_{j}, T_{j+1} \rrbracket$ then the value of the portfolio is easily seen to be described by the process $(H \cdot S)$. The final value of the process $(H \cdot S)_{\infty}$ is then given by $(H \cdot S)_{\infty}=\lim _{t \rightarrow \infty}(H \cdot S)_{t}=(H \cdot S)_{T_{n+1}}$. Stochastic integration is a natural tool in financial modeling. Predictable processes serve as strategies.

Definition 2.3 A càdlàg adapted process $S: \mathbb{R}_{+} \times \Omega \rightarrow \mathbb{R}^{d}$ is a semimartingale if for every $T<\infty$ the mapping $\mathfrak{S}_{\infty} \rightarrow L_{0}\left(\Omega, \mathcal{F}_{\infty}, \mathbb{P}\right) ; H \rightarrow$ $(H \cdot S)_{T}$ is continuous for the sup-norm topology on $\mathfrak{S}_{\infty}$. This means that if $\sup _{t, \omega}\left|H_{t}^{n}(\omega)\right| \rightarrow 0$ then $\left(H^{n} \cdot S\right)_{T} \rightarrow 0$ in probability.

If $S$ is a semi-martingale then the notion of stochastic integral $(H \cdot S)$ can be extended to all bounded predictable integrals $H$. This is the subject of the general stochastic integration theory, see e.g. [DM80], [J79], [P90].

Exactly as in the deterministic, one-dimensional case, the notion of integral can be extended to some unbounded integrands $H$. See [J79], [P90], [RW87] for details. If $(H \cdot S)$ can be defined, then we say that $H$ is $S$-integrable. Integrands that are $S$-integrable can be seen as general strategies. If the limit $(H \cdot S)_{\infty}=\lim _{t \rightarrow \infty}(H \cdot S)_{t}$ exists, then we can say that $(H \cdot S)_{\infty}$ is the final outcome of the strategy $H$.

As observed in [HK79] not all $S$-integrable processes $H$ can be accepted as reasonable strategies (from the financial point of view). Indeed we should avoid so-called doubling strategies. The most classical example of such a doubling strategy can be constructed using Rademacher functions.

Example 2.4 of a doubling strategy (discrete time) On $\left(\Omega, \mathcal{F}_{\infty}, \mathbb{P}\right)$ we take $\left(\epsilon_{n}\right)_{n \geq 1}$ to be a sequence of independent variables, so that $\mathbb{P}\left[\epsilon_{n}= \pm 1\right]=\frac{1}{2}$ 
which we may think of as the gain or loss of a risky investment revealed at time $n$. As filtration we take $\left(\mathcal{F}_{n}\right)_{n \geq 0}$ where $\mathcal{F}_{n}=\sigma\left(\epsilon_{1}, \cdots, \epsilon_{n}\right) . S_{n}$ is defined as $\epsilon_{1}+\cdots+\epsilon_{n}$. The reader can easily change the definition in such a way that $\left(\mathcal{F}_{t}\right)_{0 \leq t}$ is defined and such that the usual hypotheses hold. If a gambler buys $a$ units of the risky investment modeled by $S_{n}$ at time $n$ then his/her gain at time $n$ is given by $a \epsilon_{n}$. Let now $T=\inf \left\{n \mid \epsilon_{n}=+1\right\}$. Clearly $T<\infty$ a.s.. The doubling strategy is described by $H_{0}=1, H_{k}=2^{k}$ if $k<T$. So $f_{0}=1$ and $f_{n}=2^{n}$ if $\epsilon_{1}=\cdots=\epsilon_{n-1}=-1$. The final gain is easily calculated to be $2^{n}-\left(1+\cdots+2^{n-1}\right)=1$ if $T=n$. So we get $(H \cdot S)_{\infty}=1$ almost surely and we obtain a sure profit (in the almost sure sense, common in probability theory). But, as everybody knows, this strategy does not work in practice to make a sure profit, i.e., an arbitrage. The problem is that we may have to wait quite long before we get our first hit (i.e., before $\epsilon_{n}=+1$ for the first time). With probability $2^{-n}$ we have to wait until time $n$. In this case the gain accumulated up to time $n-1$ was given by $-\left(2^{n}-1\right)$ which can be more negative than our budget allows.

Example 2.5 of a doubling strategy (continuous time) In this example we take $S=W$ a Wiener process, i.e., Brownian motion, with its standard filtration $\left(\mathcal{F}_{t}\right)_{0 \leq t}$. The process $L_{t}=\exp \left(W_{t}-\frac{1}{2} t\right)$ is easily seen to be the unique solution of the stochastic differential equation $d L_{t}=L_{t} d W_{t}$, with initial condition $L_{0}=1$. Hence we find that

$$
1-L_{t}=\int_{] 0, t]}\left(-L_{u}\right) d W_{u}=(H \cdot W)_{t}
$$

We have that $L_{t} \rightarrow 0$ a.s. as $t \rightarrow \infty$, and therefore again $(H \cdot W)_{\infty}=1$ (while, of course, $\left.(H \cdot W)_{0}=0\right)$.

In both the discrete and the continuous example, we get that $(H \cdot S)$ is a martingale but not a uniformly integrable martingale. To exclude the phenomenon described by the above examples (where in both cases it was crucial that the process $(H \cdot S)_{t}$ was not uniformly bounded from below) one may adopt the following concept:

Definition 2.6 ([HP81] and [DS94]) An S-integrable predictable process $H$ : $\mathbb{R}_{+} \times \Omega \rightarrow \mathbb{R}^{d}$ is called a-admissible for some $a \geq 0$ if,

(i) $(H \cdot S) \geq-a$ i.e., the process always remains above the ("budget") level $-a$,

(ii) $\lim _{t \rightarrow \infty}(H \cdot S)_{t}=(H \cdot S)_{\infty}$ exists.

We call $H$ admissible if it is admissible for some $a \geq 0$. 
It is clear that the set of $a$-admissible integrands forms a cone but not necessarily a vector space.

We define:

$$
\begin{aligned}
K & =\left\{(H \cdot S)_{\infty} \mid H \text { admissible }\right\} \\
\mathcal{C} & =\left\{k-h \mid k \in K, h \in L_{0}^{+}\right\} \cap L_{\infty}=\left(K-L_{0}^{+}\right) \cap L_{\infty}
\end{aligned}
$$

The set $K$ has an easy interpretation, it is simply the cone of all outcomes of admissible strategies (starting with an initial wealth zero). The set $\mathcal{C}$ has a similar interpretation. It is a philanthropic version of $K$ in the sense that after having obtained $(H \cdot S)_{\infty}$ the investor can give away a nonnegative amount of money thus making the gain uniformly bounded. Before continuing, let us point out that in the case of non-locally bounded processes it may happen that $K$ is reduced to $\{0\}$. Indeed, for an easy example take $S_{t}=0$ for $t<1$ and $S_{t}=X$ for $t \geq 1$ where $X$ is a standard normal variable. The filtration $\left(\mathcal{F}_{t}\right)_{0 \leq t}$ is the filtration generated by $\mathrm{S}$. For a predictable integrand $H$ we have that $H_{1}=$ constant. It follows that $(H \cdot S)_{\infty}=(H \cdot S)_{1}$ is of the form $\lambda X$ where $\lambda \in \mathbb{R}$ and $H$ is only admissible if $H_{1}=0$ which yields $(H \cdot S)_{\infty}=0$. In this example we obtain that $\mathcal{C}=-L_{\infty}^{+}$.

We now have enough material to define the No Arbitrage properties. Roughly speaking these properties say that it is impossible to make money out of nothing (no money pump, no arbitrage, no free lunch...). Because of doubling strategies we have to restrict to admissible integrands.

Definition 2.7 ([DS94]) The semi-martingale $S$ satisfies the No Arbitrage (NA) property if $K \cap L_{0}^{+}=\{0\}$ or, equivalently, $\mathcal{C} \cap L_{0}^{+}=\{0\}$.

The idea of this notion is that, by using admissible strategies, the investor can only make money if he/she is willing to face the possibility of also losing money. A standard and very intuitive example of $(N A)$ is given by:

Proposition 2.8 If there is a probability measure $\mathbb{Q} \sim \mathbb{P}$ under which $S$ is a martingale, then $S$ satisfies (NA).

Proof Let $H$ be admissible and $\mathbb{Q}$ a probability measure, $\mathbb{Q} \sim \mathbb{P}$; then $(H \cdot S)$ is a $\mathbb{Q}$-local martingale by Emery's theorem (see $[\mathrm{E} 80])$. Since $(H \cdot S)$ is bounded below it is a $\mathbb{Q}$-super martingale and hence $\mathbb{E}_{\mathbb{Q}}\left[(H \cdot S)_{\infty}\right] \leq 0$. It follows from the assumption $\mathbb{Q} \sim \mathbb{P}$ that $\mathbb{P}\left[(H \cdot S)_{\infty}<0\right]>0$ as soon as $\mathbb{P}\left[(H \cdot S)_{\infty}>0\right]>0$ i.e., $(N A)$.

The fundamental theorem of asset pricing is the appropriate converse of proposition 2.8. It is easily seen that in the case of a finite set $\Omega$, the cone $K$ is a vector space and an easy separation argument yields the existence of a risk neutral probability $\mathbb{Q}$ such that $S$ becomes a martingale (compare the 
more general arguments below). However in the general case, it is not clear that the sets $K$ or $\mathcal{C}$ are closed (in an appropriate topology). Also in a general context it can be shown that it is impossible to obtain a martingale measure $\mathbb{Q}$ for a process $S$. This is of course related to the integrability properties of the process $S$. The generalizations we need are described in the following definition.

Definition 2.9 If $\left(\Omega,\left(\mathcal{F}_{t}\right)_{0 \leq t}, \mathbb{P}\right)$ is a filtered probability space, then a process $S: \mathbb{R}_{+} \times \Omega \rightarrow \mathbb{R}$ (or $\mathbb{R}^{d}$ ) is called a local martingale if there is a non-decreasing sequence of stopping times $0 \leq T_{0} \leq T_{1} \leq T_{2} \leq \ldots$ such that $T_{n} \rightarrow \infty$ and for each $n$ we have that the stopped process $S^{T_{n}}$ is a uniformly integrable martingale. We recall that $S^{T}$ is defined as the process $S_{t}^{T}=S_{t \wedge T}$.

In case the time interval is finite, e.g. $[0,1]$, we require that $\lim \mathbb{P}\left[T_{n}=\right.$ $1]=1$.

A standard example of a uniformly integrable (!) process $S$ that is a local martingale and not a martingale is given by the inverse of a Bessel process in 3 dimensions (see, e.g., [RY91]). The process is defined as follows. We start with a 3 -dimensional Brownian motion $X:[0,1] \times \Omega \rightarrow \mathbb{R}^{3}$. The filtration is the standard filtration generated by $X$. We suppose that $X_{0}=(1,0,0)$. The Bessel process $R$ is defined as $R=\|X\|$ where \|\| is the Euclidean norm on $\mathbb{R}^{3}$. By the well known property of Brownian motion in 3 dimensions we have that $R \neq 0$ and $S=\frac{1}{R}$ is therefore well defined. It can be checked that $S$ is a local martingale (roughly speaking because $\frac{1}{\|R\|}$ is harmonic on $\mathbb{R}^{3} \backslash\{(0,0,0)\}$ ), that $\left(S_{t}\right)_{0 \leq t \leq 1}$ is a uniformly integrable set (in fact bounded in any $L_{p}$ space with $p<3$ ) and that $\mathbb{E}\left[S_{t}\right]<1$. The latter shows that $S$ cannot be a martingale. If we replace the filtration by the smaller filtration generated by $S$, then it can be shown that $\mathbb{P}$ is the only probability that turns $S$ into a local martingale. The reader can check that proposition 2.8 also holds for local martingales and hence we get that $S$ satisfies (NA) (see [RY91] for details on Bessel process).

For non continuous processes the concept of local martingale is not yet sufficient as is shown in [DS98]. We need the even more general concept of sigma-martingale. These processes were introduced by Chou and Emery (see $[\mathrm{E} 80])$.

Definition 2.10 ([DS98]) A process $S: \mathbb{R}_{+} \times \Omega \rightarrow \mathbb{R}$ defined on the filtered probability space $\left(\Omega,\left(\mathcal{F}_{t}\right)_{0 \leq t}, \mathbb{P}\right)$ is called a sigma-martingale if there is a strictly positive predictable process $\varphi: \mathbb{R}_{+} \times \Omega \rightarrow \mathbb{R}_{+} \backslash\{0\}$ such that $\varphi \cdot S$ is a uniformly integrable martingale.

It is easily seen that a local martingale is a sigma-martingale. Conversely if in the above definition the function $\varphi$ can in addition be chosen to be decreasing then the sigma-martingale is already a local martingale. For continuous (or, more generally, for locally bounded) processes the two concepts coincide. 
In the usual models of mathematical finance it does not matter if we work with the process $S$ or with a process $\varphi \cdot S$ where $\varphi$ is non-zero. Indeed our main concern goes to stochastic integrals and it is obvious that for predictable processes $H: \mathbb{R}_{+} \times \Omega \rightarrow \mathbb{R}^{d}$ we have that $H$ is $(\varphi \cdot S)$ integrable if and only if $(H \varphi)$ is $S$ integrable. As a result we see that the sets $\mathcal{C}$ and $K$ defined by $S$ or by $\varphi \cdot S$ are the same.

\section{The Fundamental Theorem of Asset Pricing}

We start this section with a generalization of the concept of No Arbitrage, which can be seen as a topological version of $(N A)$.

Definition 3.1 With the notation of section 2 we say that the semi-martingale $S: \mathbb{R}_{+} \times \Omega \rightarrow \mathbb{R}^{d}$ satisfies the No Free Lunch with Vanishing Risk (NFLVR) property if $\overline{\mathcal{C}} \cap L_{\infty}^{+}=\{0\}$ where $\overline{\mathcal{C}}$ is the closure of $\mathcal{C}$ in $L_{\infty}$ with respect to the norm \|\|$_{\infty}$.

There are different topological versions of (NA) pertaining to weaker topologies than the sup-norm (and therefore yielding stronger assumptions). We refer the reader to [DS94] for a discussion of the different concepts.

Theorem 3.2 A semi-martingale $S$ satisfies (NFLVR) if and only if $S$ satisfies the following two properties

(a) $S$ satisfies (NA)

(b) If $\epsilon_{n}>0$ is a sequence of positive numbers tending to zero, if $\left(H^{n}\right)_{n \geq 1}$ is a sequence of $\epsilon_{n}$-admissible strategies (i.e., $H^{n} \cdot S \geq$ $\left.-\epsilon_{n}\right)$ then $\left(H^{n} \cdot S\right)_{\infty} \rightarrow 0$ in probability.

One may give examples showing that (a) does not imply (b) and (b) does not imply (a). For the former we defer the discussion to section 4 below. For the latter we may take the Bessel process $R$ in three dimensions (see [DS95]). We drop the details. The reader can now see why the property is called No Free Lunch with Vanishing Risk.

The No Free Lunch with Vanishing Risk property is also equivalent to a boundedness property. In fact we have:

Theorem 3.3 A semi-martingale $S$ satisfies (NFLVR) if and only if $S$ satisfies the following two properties

(a) $S$ satisfies (NA)

(b) The set $K=\left\{(H \cdot S)_{\infty} \mid H\right.$ is 1-admissible $\}$ is bounded in probability. 
The boundedness property also has an immediate economic interpretation. If strategies are chosen so that the losses are bounded uniformly (i.e., in $L_{\infty}$ ), then the gains are bounded in probability (i.e., in $L_{0}$ ). This can also be seen as some continuity property. Of course, the relation between items (b) of the two preceding theorems is immediately noticeable.

The fundamental theorem of asset pricing in its most general form can now be stated (see [DS94] and [DS98]).

Theorem 3.4 For a semi-martingale $S: \mathbb{R}_{+} \times \Omega \rightarrow \mathbb{R}^{d}$ the following two properties are equivalent

(a) S satisfies the (NFLVR) property

(b) There is an equivalent probability measure $\mathbb{Q} \sim P$ such that under $\mathbb{Q}$ the process $S$ is a sigma-martingale.

If we assume that the semi-martingale $S$ is locally bounded (resp. bounded) the term "sigma-martingale" in (b) may be replaced by the term "local martingale" (resp. "martingale").

In case the (NFLVR) property is satisfied we denote by $\mathbb{M}^{e}$ the set of all equivalent sigma-martingale measures. The set $\mathbb{M}$ is the set of all absolutely continuous semi-martingale measures. In case $S$ is locally bounded $\mathbb{M}=\overline{\mathbb{M} e}$, where the closure is taken with respect to the norm of $L_{1}(\mathbb{P})$, but with general case $\mathbb{M} \varsubsetneqq \overline{\mathbb{M}^{e}}$.

The proof of this theorem is lengthy and we cannot repeat it here. But we will give a sketch of the different points that relate the theorem to functional analysis. The first and most delicate step consists in proving that under the assumption of (NFLVR) the set $\mathcal{C}$ is already weak*-closed. Yan's theorem [Yan80] (whose proof consists of a combination of a Hahn-Banach and an exhaustion argument) (see, e.g., [S94]) then yields the existence of an equivalent probability measure $\mathbb{Q}$ such that, for all $f \in \mathcal{C}, \mathbb{E}_{\mathbb{Q}}[f] \leq 0$. Interpreting $\mathbb{Q}$ as a linear functional on $L_{\infty}$, it separates $\mathcal{C}$ from the positive cone $L_{\infty}^{+}$. In the case where $S$ is locally bounded this already implies that $S$ is a local martingale for the measure $\mathbb{Q}$. In the general case one has to make an additional (nontrivial) step that consists in showing that, for $\epsilon>0$, there is a measure $\mathbb{Q}_{0}$ such that $\mathbb{Q}_{0}$ is equivalent to $\mathbb{P}$, that $\left\|\mathbb{Q}-\mathbb{Q}_{0}\right\| \leq \epsilon$ and that under $\mathbb{Q}_{0}$ the process $S$ is a sigma-martingale. See [DS98] for details and for an example that shows that one cannot do better than a sigma-martingale.

As indicated above, the central point of the proof is the fact that $\mathcal{C}$ is weak*-closed. This is done using the Banach-Diendonné or - what amounts essentially to the same - the Krein-Smulian theorem. This theorem says that a convex set $C$ in the dual $X^{*}$ of a Banach space $X$ is $\sigma\left(X^{*}, X\right)$ (i.e., weak*closed) if and only if $C \cap\left(n B_{X^{*}}\right)$ is $\sigma\left(X^{*}, X\right)$ closed for each $n \geq 1$. If $X=L_{1}$ and $X^{*}=L_{\infty}$ we can, using the characterization of relatively weakly compact sets in $L_{1}$ as uniformly integrable subsets of $L_{1}$, make this even more precise. 
A convex set $C \subset L_{\infty}$ is weak*-closed if and only if, for each sequence $\left(f_{n}\right)_{n \geq 1}$ in $C$ that is uniformly bounded and converges in probability to a function $f$, we have that $f \in C$. Since in our context the set $\mathcal{C}$ is a cone we have to show the following fact.

Claim 3.5 Let $\left(H^{n}\right)_{n \geq 1}$ be a sequence of 1-admissible integrands, let $\left(f_{n}\right)_{n \geq 1}$ be a sequence in $L^{0}(\Omega, \mathcal{F}, \mathbb{P})$ such that $-1 \leq f_{n} \leq\left(H^{n} \cdot S\right)_{\infty}$, which tends to $f$ in probability; then under the assumption of (NFLVR) there is a 1-admissible integrand $H$ such that $f \leq(H \cdot S)_{\infty}$.

To prove this claim we have to replace the integrands by a better choice. Let us define the following concept:

Definition 3.6 An element $f \in \overline{\mathcal{C}}_{1}$ is called maximal if $g \in \overline{\mathcal{C}}$ and $g \geq f$ a.s. implies $g=f$. Here $\mathcal{C}_{1}$ denotes the set $\mathcal{C}_{1}=\left\{h \mid h \in \mathcal{C}_{0} \quad h \geq-1\right\}$ and the bar refers to the closure in the space $L_{0}$ (i.e., with respect to convergence in probability).

Our assumptions on (NFLVR) show that $\mathcal{C}_{1}$ is bounded in $L_{0}$ and hence $\overline{\mathcal{C}}_{1}$ has sufficiently many maximal elements. In fact every $g \in \overline{\mathcal{C}}_{1}$ is dominated by such a maximal element. To prove the claim we reduce it to the following:

Claim 3.7 The maximal elements of $\overline{\mathcal{C}}_{1}$ are already in $\mathcal{C}_{1}$.

So let us fix a sequence $\left(H^{n}\right)_{n \geq 1}$ such that $H^{n}$ is 1-admissible and such that $\left(H^{n} \cdot S\right)_{\infty} \rightarrow f$ in probability, where $f$ is a maximal element in $\overline{\mathcal{C}}_{1}$. The first trick is to replace the probability $\mathbb{P}$ by an equivalent probability (still denoted by $\mathbb{P}$ ) such that the function $\sup _{n \geq 1} \sup _{t}\left|\left(H^{n} \cdot S\right)_{t}\right|$ is in $L_{2}(\mathbb{P})$. That this is possible follows from an upcrossing type lemma and the maximality of $f$. After an additional technical reduction, which we skip, the Doob-Meyer decomposition theorem now allows to decompose $S$ into a martingale $M$ and a predictable process of finite variation $A$, i.e., $S=M+A$. The Doob-Meyer decompositions of $\left(H^{n} \cdot S\right)$ are then given by $\left(H^{n} \cdot M\right)$ and $\left(H^{n} \cdot A\right)$. In order to control the jumps of $M$ and $A$ we use the following generalization of an inequality of Stein.

Proposition 3.8 Let $\left(\mathcal{F}_{n}\right)_{n=0, \ldots, N}$ be a discrete time filtration on the probability space $(\Omega, \mathcal{F}, \mathbb{P})$, let $\left(f_{n}\right)_{n=1, \ldots, N}$ be adapted to $\left(\mathcal{F}_{n}\right)_{n=0, \ldots, N}$, let $g_{n}$ be the predictable projection, i.e., $g_{n}=\mathbb{E}\left[f_{n} \mid \mathcal{F}_{n-1}\right]$ then we have

$$
\mathbb{E}\left[\left(\sum\left|g_{n}\right|^{q}\right)^{\frac{p}{q}}\right]^{\frac{1}{p}} \leq 2 \mathbb{E}\left[\left(\sum\left|f_{n}\right|^{q}\right)^{\frac{p}{q}}\right]^{\frac{1}{p}}
$$

whenever $1 \leq p \leq q \leq \infty$. In other words the mapping

$$
\begin{aligned}
L^{p}\left(l_{q}^{N}\right) & \longrightarrow L^{p}\left(l_{q}^{N}\right) \\
\left(f_{n}\right)_{n \geq 1} & \longmapsto\left(\mathbb{E}\left[f_{n} \mid \mathcal{F}_{n-1}\right]\right)_{n \geq 1}
\end{aligned}
$$


has norm less than or equal to 2.

The next step is to show that the convex hull of $\left(\left(H^{n} \cdot M\right)_{\infty}\right)_{n \geq 1}$ and of $\left(\left(H^{n} \cdot A\right)_{\infty}\right)_{n \geq 1}$ are both bounded (in a good sense). Since $\left(\left(H^{n} \cdot S\right)_{\infty}\right)_{n \geq 1}$ is bounded in $L_{2}(\mathbb{P})$ both sets are at the same time bounded or unbounded. But if they are unbounded we are faced with the fact that $\left(H^{n} \cdot A\right)_{t}$ increases in a "linear" way whereas $\left(H^{n} \cdot M\right)_{t}$, due to the orthogonality of its increments, grows only in a way related to the square root. This leads to a contradiction. Once the boundedness is proved, it is a straightforward track to find convex combinations $K^{n}$ of $\left(H^{n}\right)_{n \geq 1}$ that converge to a predictable process $K$ such that $(K \cdot S)_{\infty}=f$. The latter technique is a combination of a Hahn-Banach type argument together with the techniques of Memin (see [M80]).

The separation argument in the proof of the fundamental theorem can be exploited further. It yields to the following duality result (see [EQ95], [D92]).

Theorem 3.9 Assume that $S$ is a locally bounded semi-martingale such that $\mathbb{M}^{e}$ is not empty. Then for $f \geq 0$ we get

$$
\sup _{\mathbb{Q} \in \mathbb{M}^{e}} \mathbb{E}_{\mathbb{Q}}[f]=\inf \{\alpha \mid \exists g \in K \text { with } \alpha+g \geq f\}=: \alpha_{0}
$$

Furthermore, if the quantities are finite, the infimum is a minimum and there is a maximal element $g \in K^{\max }$ with $\alpha_{0}+g \geq f$.

\section{The continuous case}

When the process $S$ is continuous or, more generally, locally bounded, the assumption on $S$ to be a semi-martingale turns out to be a necessary condition for the conclusion of the fundamental theorem of asset pricing to hold true. In fact we can prove the following result (see [DS94]), stated under a finite time horizon, say $[0,1]$.

Theorem 4.1 If the locally bounded process $S=\left(S_{t}\right)_{0 \leq t \leq 1}$ is not a semimartingale then there is a function $f \geq 0, \mathbb{P}[f>0]>0$, and a sequence of simple strategies $H_{n}$ such that $\left(H_{n} \cdot S\right) \geq-\epsilon_{n}, \epsilon_{n} \rightarrow 0,\left(H_{n} \cdot S\right)_{1} \stackrel{\mathbb{P}}{\rightarrow} f$.

We recall that a strategy $H$ is simple, if there is a finite sequence of stopping times $0 \leq T_{0} \leq T_{1} \leq \ldots \leq T_{n}<\infty$ as well as functions $\left(g_{k}\right)_{k \geq 0}, g_{k}$ being $\mathcal{F}_{T_{k}}$ measurable and $H=\sum_{k=0}^{n-1} g_{k} \mathbf{1}_{\llbracket T_{k}, T_{k+1} \rrbracket}$. These strategies are most elementary and the definition of their stochastic integral $(H \cdot S)$ does not involve any calculus.

The theorem is not true for processes with unbounded jumps, since in this case the boundedness condition on $(H \cdot S)$ might imply that $H \equiv 0$.

Theorem 4.1 has some nice consequences for continuous processes. In particular fractional Brownian motion fails to be a semi-martingale (with the 
exception of Brownian motion, of course) and therefore allows, by theorem 4.1, this kind of arbitrage. This implies that these models are useless in the context of pricing and hedging derivative securities by no-arbitrage arguments. This fact was known since a long time, but even after publication of the above theorem, people still continued to ask about fractional Brownian motion. Chris Rogers [R97] decided to settle the problem by giving a fairly explicit strategy $H$ such that $(H \cdot S)_{1} \geq 0$ and $\mathbb{P}\left[(H \cdot S)_{1}>0\right]>0,(H \cdot S) \geq-1$.

The strategy is not simple (one can show that for simple strategies $(N A)$ is satisfied), but it is "semi"-simple in the sense that restricted to each interval $[0, t]$ with $t<1$, it is simple. Fractional Brownian motion, however, has some nice long range dependencies, observed in financial data. In forthcoming work of Cheridito [Ch00] the reader can find a way out to this dilemma.

For continuous processes the concept of $(N A)$ can be analyzed further. So let us assume that $S$ is a continuous semi-martingale with respect to a filtered space $\left(\Omega,\left(\mathcal{F}_{t}\right)_{0 \leq t \leq 1}, \mathbb{P}\right)$; again for simplicity we assume that the horizon is finite, say $[0,1]$. The Doob-Meyer theorem permits to write $S=M+A$ where $M$ is a continuous local martingale, $A$ is a process of finite variation with $A_{0}=0$. If $S$ satisfies (NA) then it is immediately seen that the measure $d A$ is absolutely continuous with respect to the measure $d\langle M, M\rangle$ (see the argument some lines below).

The process $\langle M, M\rangle$ is the quadratic variation of $M$ (and hence also of $S$ ). In this case we can define $\langle M, M\rangle$ as $M^{2}-2 M \cdot M$. Recall that in stochastic calculus the derivative of $M^{2}$ is not equal to $2 M d M$, it involves a second order term (Itô's lemma). This term is precisely $d\langle M, M\rangle$.

The fact that $d A$ is of the form $h d\langle M, M\rangle$ for some predictable process $h$ is then a consequence of the Radon-Nikodym theorem. In the stochastic case the standard proofs need some changes, the theory was developed by C. Doléans-Dade, see [DS95]. If $d A$ were not absolutely continuous with respect to $d\langle M, M\rangle$ then it follows from Hahn's decomposition theorem that there is a strategy $H$ such that $H=0, d\langle M, M\rangle$ a.s. and $H d A$ is strictly positive. If follows that $H \cdot M=0$ and that $H \cdot S=H \cdot A$ is an increasing process. This implies arbitrage. The strategy $H$ is roughly speaking so that only trades when the random "risky" component $M$ of $S$ does not move. In this way the agent takes advantage of the predictable finite variation part.

In arbitrage considerations we therefore may suppose that a continuous semi-martingale $S$ satisfies $d S=d M+h d\langle M, M\rangle$ where $\mathrm{M}$ is the local martingale part in the Doob-Meyer decomposition and $h$ is a predictable process. The existence of an equivalent local martingale measure for $S$ is intimately related to the process $\exp \left(-\int_{0}^{t} h_{u} d M_{u}-\frac{1}{2} \int_{0}^{t} h_{u}^{2} d\langle M, M\rangle_{u}\right)$, the so called Doléans-Dade or stochastic exponential of $(-h \cdot M)$, also written as $\mathcal{E}(-h \cdot M)$. Of course the existence depends on the finiteness of the integral $\int_{0}^{t} h_{u}^{2} d\langle M, M\rangle_{u}$. If the integral is not almost surely finite then there are essentially two possibilities. 
To distinguish them we introduce the stopping time

$$
T=\inf \left\{t \mid \int_{0}^{t} h_{u}^{2} d\langle M, M\rangle_{u}=\infty\right\} .
$$

On the set $T \leq 1$ we then have either $\int_{0}^{T} h_{u}^{2} d\langle M, M\rangle_{u}=\infty$ or we have, $\forall \epsilon>0$, $\int_{T}^{T+\epsilon} h_{u}^{2} d\langle M, M\rangle_{u}=\infty$.

Technically the latter case is more difficult but it has a simple solution: there is a very special kind of arbitrage, namely there is $H$ supported by $\rrbracket T, 1 \rrbracket$ and such that

$(H \cdot S) \geq 0$ i.e., the outcome at every time is nonnegative, and $\mathbb{P}\left[(H \cdot S)_{T+\epsilon}>0\right]>0$ for all $\epsilon>0$.

An example of this nature is given by $d S_{t}=\frac{d t}{\sqrt{t}}+d W_{t}$ where $W$ is Brownian motion defined in its natural filtered space. $\AA^{t}$ strategy of the form above is given by $K_{t}=\frac{1}{\sqrt{t} \log \left(t^{-1}\right)}$ and then we stop the process $(K \cdot S)$ either when it reaches a level 1 or when it hits 0 for the first time after 0 . The iterated logarithm theorem implies that, immediately after 0 the process $(K \cdot S)$ is strictly positive!

The next result is closely related to these arguments. It was shown in [DS95] and, independently and under slightly stronger hypothesis, in [LS95].

Theorem 4.2 If the continuous martingale $S$ satisfies (NA) then there is a measure $\mathbb{Q}$ absolutely continuous with respect to $\mathbb{P}$ and such that $S$ is a local martingale under $\mathbb{Q}$. The support of the measure $\mathbb{Q}$ can be chosen to be equal to $\left\{L_{1}>0\right\}$ where $L_{t}=\exp \left(-\int_{0}^{t} h_{u} d M_{u}-\frac{1}{2} \int_{0}^{t} h_{u}^{2} d\langle M, M\rangle_{u}\right)$ stopped when $\int_{0}^{t} h_{u}^{2} d\langle M, M\rangle_{u}$ hits $\infty$, or what is the same: when $L$ hits 0 .

Even when $S$ satisfies (NA) and $\int_{0}^{1} h_{u}^{2} d\langle M, M\rangle_{u}<\infty$ a.s., the process $L$ need not be a martingale, i.e., it can happen that $E\left[L_{1}\right]<1$. This means that the measure $\mathbb{Q}$ is not necessarily given by $d S=L_{1} d \mathbb{P}$. See [S93] and [DS98a].

We also have the following:

Proposition 4.3 If $S$ is a continuous semi-martingale decomposed as $d S=$ $d M+h d\langle M, M\rangle$ then the set $K_{1}=\left\{(H \cdot S)_{\infty} \mid H\right.$ is 1-admissible $\}$ is bounded in $L_{0}(\mathbb{P})$ if and only if $\int_{0}^{1} h_{u}^{2} d\langle M, M\rangle_{u}<\infty$ a.s.

The 3-dimensional Bessel process shows that this does not rule out arbitrage (compare [DS95a])!

\section{Changes of Numéraire and a related Banach space}

Throughout this section we suppose that the process $S$ modeling the price of $d$ stocks is a $d$-dimensional semi-martingale that is locally bounded. We also 
suppose that the process $S$ admits a local martingale measure. We repeat that the set of equivalent local martingale measures is denoted by $\mathbb{M}^{e}$, the notation $\mathbb{M}$ being reserved for the closed convex set (in fact the closure of $\mathbb{M}^{e}$ ) of absolutely continuous local martingale measures for $S$. $K_{a}$ will denote the cone of outcomes of ( $a$-)admissible strategies, $K=\bigcup_{a} K_{a}$. As seen before, maximal elements play an important role in the proof of the fundamental theorem. However, more can be said. If $V=1+(H \cdot S)$ is a strictly positive process satisfying $V_{\infty}>0$ a.s., then we might use $V$ as a new money unit (say $\$$ instead of CHF or Euro). The market is now described by the $(d+1)$-dimensional process $X=\left(\frac{1}{V}, \frac{S}{V}\right)$. Economic interpretation leads us to conjecture that $S$ satisfies (NA) if and only if $X$ satisfies $(N A)$ : It does not matter whether you do the bookkeeping in $\$, \mathrm{CHF}$ or in Euro! However since the admissible strategies may change, we have to be more careful.

Theorem 5.1 If $S$ satisfies (NFLVR) then the following assertions are equivalent for a process $V=1+(H \cdot S)$, where $H$ is admissible and $V_{\infty}>0$ a.s.:

(1) $X=\left(\frac{1}{V}, \frac{S}{V}\right)$ satisfies (NA),

(2) there is an equivalent local martingale $\mathbb{Q}$ measure for the process $S$, such that $V$ is a uniformly integrable $\mathbb{Q}$-martingale, i.e., $\mathbb{E}_{\mathbb{Q}}\left[V_{\infty}\right]=1$ or equivalently $\mathbb{E}_{\mathbb{Q}}\left[(H \cdot S)_{\infty}\right]=0$,

$(3)(H \cdot S)_{\infty}$ is maximal in $K_{1}$ (and hence also in $K$ ).

It is not true that in this case $V$ is a uniformly integrable martingale for each element $R$ in the set of equivalent local martingale measures $\mathbb{M}^{e}$, see [S93] or [DS98a] for this surprising fact. The above theorem also yields another proof of the theorems of Jacka [J92] and Ansel-Stricker [AS94].

Theorem 5.2 If $S$ satisfies (NFLVR) and $f$ is a positive random variable, then the following conditions are equivalent:

(a) $f=\alpha+(H \cdot S)_{\infty}$ with $(H \cdot S)_{\infty}$ maximal in $K$

(b) there is $\mathbb{Q} \in \mathbb{M}^{e}$ such that $\mathbb{E}_{\mathbb{Q}}[f]=\sup \left\{\mathbb{E}_{R}[f] \mid R \in \mathbb{M}\right\}<\infty$

The Bishop-Phelps theorem now immediately implies

Theorem 5.3 If S satisfies (NFLVR) and all absolutely continuous local martingale measures are already equivalent, then $\mathbb{M}$ is reduced to a singleton.

Proof As the set of absolutely continuous local martingale measures $\mathbb{M}$ is a bounded, closed and convex subset of $L_{1}$, the set $\{f \mid f$ attains its supremum on $\mathbb{M}\}$ is a norm dense subset of $L_{\infty}$. The set of all elements of the form $\alpha+(H \cdot S)_{\infty}$, where the process $(H \cdot S)$ is bounded is therefore dense in $L_{\infty}$ and because it is closed (this follows essentially from claim 3.5) we have that it equals $L_{\infty}$. This implies that all elements of $L_{\infty}$ are 
constant on $\mathbb{M}$, hence $\mathbb{M}$ can only be a one-point-set.

Another application of Banach space theory is given by James' theorem on weakly compact sets. We state the result in its negative form, see [D92] for details.

Theorem 5.4 Suppose $S$ is continuous, satisfies (NFLVR) and suppose that all martingales with respect to $\left(\mathcal{F}_{t}\right)_{0 \leq t}$ are continuous (i.e., all stopping times are predictable). Then we have

(a) either $\mathbb{M}$ is a singleton

(b) or $\mathbb{M}$ is so big that it has no extreme points.

It turns out that (a) occurs if and only if $\mathbb{M}$ is weakly compact and the proof uses James' theorem.

In the case when $S$ is only assumed to be locally bounded (and not necessarily continuous), the above theorem is false and the implications of $\mathbb{M}$ being weakly compact are not yet fully understood.

Turning back to the theme of theorem 5.1 above, we find that the maximal elements generate, in a natural way, a Banach space.

Theorem 5.5 The set $K^{\max }$ of maximal elements forms a cone in $L^{1}(\mathbb{P})$.

This is not obvious. Indeed if $g, f \in K, \mathbb{E}_{\mathbb{Q}}[f]=0$ and $\mathbb{E}_{R}[g]=0$ for $\mathbb{Q}, R \in \mathbb{M}^{e}$ then how can we find an element $\mathbb{Q}^{\prime} \in \mathbb{M}^{e}$ with $\mathbb{E}_{\mathbb{Q}^{\prime}}[f+g]=0$ ? The proof uses the numeraire theorem 5.1 above.

With the convex cone $K^{\max }$ we may construct the vector space $G=K^{\text {max }}$ $K^{\max }$. On $G$ there is natural norm, if $g=f-h$ where $f, h \in K^{\max }$ then we put

$$
\|g\|=\inf \left\{a \mid g=f-h, f, h \in K_{a}^{\max }\right\} .
$$

Surprisingly $(G,\|\|)$ is complete and

$$
2\|g\|=\sup \left\{\|g\|_{L_{1}(\mathbb{Q})} \mid \mathbb{Q} \in \mathbb{M}^{e}\right\} .
$$

Although the constructions of $K^{\text {max }}$ and of $G$ make sense economically, the implications of this result in mathematical finance are not yet clear. Examples in [DS97] show that the space $G$ can have different natures, it can be an $L_{1}$ space but it can also contain a complemented $L_{\infty}$ space. Also the space $G$ is different from the space of functions $f$ so that $\sup \left\{\|f\|_{L_{1}(\mathbb{Q})} \mid \mathbb{Q} \in \mathbb{M}^{e}\right\}<\infty$. Indeed take $f \in K_{1} \backslash K^{\max }$ (such elements exist) then $f \notin G$ (this requires a proof!) and $\sup _{\mathbb{Q} \in \mathbb{M}^{e}}\|f\|_{L_{1}(\mathbb{Q})} \leq 1$.

A side result of the theory is the following 
Theorem 5.6 If $f \in K^{\max }$ then

$$
\left\{\mathbb{Q} \mid \mathbb{Q} \in \mathbb{M}, \mathbb{E}_{\mathbb{Q}}[f]=0\right\}
$$

is a dense $G_{\delta^{-}}$set in $\mathbb{M}$. Hence $\left\{\mathbb{Q} \mid \mathbb{Q} \in \mathbb{M}^{e}, \mathbb{E}_{\mathbb{Q}}[f]=0\right\}$ is a dense $G_{\delta^{-}}$set in $\mathbb{M}^{e}$.

\section{$6 \quad$ Weighted Norm Inequalities and Closedness of a Space of Stochastic Integrals}

In this section we investigate a topic initiated by the work of FöllmerSondermann [FS86] and Föllmer-Schweizer [FSch91], to deal with the problem of hedging in incomplete markets, i.e., when there is no uniqueness of the equivalent martingale measure.

To motivate the idea, first suppose for simplicity that $S$ is an $\mathbb{R}$-valued martingale under the original measure $\mathbb{P}$ which we also assume to be bounded in $L^{2}(\Omega, \mathcal{F}, \mathbb{P})$. We denote by $G$ the subspace of $L^{2}(\Omega, \mathcal{F}, \mathbb{P})$ consisting of the functions of the form

$$
\begin{aligned}
f & =\int_{0}^{T} H_{u} d S_{u} \\
& =(H \cdot S)_{T}
\end{aligned}
$$

where $H$ is a predictable process such that the stochastic integral makes sense in $L^{2}$, i.e., such that $\left((H \cdot S)_{t}\right)_{t \leq T}$ is a martingale bounded in $L^{2}(\Omega, \mathcal{F}, \mathbb{P})$. In this setting it is an easy consequence of the very definition of a stochastic integral that $G$ is a closed subspace of $L^{2}(\Omega, \mathcal{F}, \mathbb{P})$ : indeed, we have the KunitaWabanabe isometry

$$
\begin{aligned}
\left\|\int_{0}^{T} H_{u} d S_{u}\right\|_{L^{2}(\Omega, \mathcal{F}, \mathbb{P})} & \left.=\|H\|_{L^{2}(\Omega \times[0, T], \mathcal{P}, d \mathbb{P} d\langle S\rangle}\right) \\
& =\left[\int_{\Omega} \int_{0}^{T} H_{t}^{2}(\omega) d\langle S\rangle_{t}(\omega) d \mathbb{P}\right]^{\frac{1}{2}} .
\end{aligned}
$$

Here $\mathcal{P}$ denotes the sigma-algebra of predictable subsets of $\Omega \times[0, T]$ and $d \mathbb{P} d\langle S\rangle$ denotes the finite measure on $\mathcal{P}$ induced by the quadratic variation process of $S$. As the space $L^{2}(\Omega \times[0, T], \mathcal{P}, d \mathbb{P} d\langle S\rangle)$ is obviously complete and can be identified via the above isometry with the subspace $G$ of $L^{2}(\Omega, \mathcal{F}, \mathbb{P})$ we find that $G$ is closed in $L^{2}(\Omega, \mathcal{F}, \mathbb{P})$.

We denote by $\widetilde{G}$ the space spanned by $G$ and the constant functions which clearly again is closed in $L^{2}$. Note in passing that in the case when $\left(S_{t}\right)_{0 \leq t \leq T}$ is Brownian motion defined on its natural filtration $\left(\mathcal{F}_{t}\right)_{0 \leq t \leq T}$ then $\widetilde{G}$ equals the 
entire space $L^{2}(\Omega, \mathcal{F}, \mathbb{P})$; this corresponds to the $L^{2}$-version of the martingale representation theorem for Brownian motion mentioned in the introduction.

As $\widetilde{G}$ is closed, we may define the orthogonal projection $\pi_{\widetilde{G}}: L^{2}(\Omega, \mathcal{F}, \mathbb{P}) \mapsto$ $\widetilde{G}$. Given a contingent claim $f \in L^{2}(\Omega, \mathcal{F}, \mathbb{P})$ we now may, following FöllmerSondermann [FS86], decompose $f$ into

$$
f=c+g+h
$$

where $c$ is a constant, $g$ may be written as $g=(H \cdot S)_{T}$ while $h$ is orthogonal to $\widetilde{G}$. We may associate to $g$ and $h$ the martingales $\mathbb{E}\left[g \mid \mathcal{F}_{t}\right]_{0 \leq t \leq T}=\left((H \cdot S)_{t}\right)_{0 \leq t \leq T}$ and $\left(\mathbb{E}\left[h \mid \mathcal{F}_{t}\right]\right)_{0 \leq t \leq T}$, the latter martingale being strongly orthogonal to the martingale $\left(S_{t}\right)_{0 \leq t \leq T}$

The interpretation in Finance goes as follows: a general contingent claim $f$ may be decomposed into the "hedgeable" part $c+g$ which may be replicated at an initial price $c$; the remaining part is given by the "purely non-hedgeable" risk $h$.

So far we have used the simplifying assumption that the price process $S=\left(S_{t}\right)_{0 \leq t \leq T}$ is already a martingale. We now turn to the case when $S$ is only an $\mathbb{R}^{d}$-valued semi-martingale; following Föllmer-Schweizer [FSch91] our aim is to decompose a general contingent claim $f \in L^{2}(\Omega, \mathcal{F}, \mathbb{P})$ into a hedgeable and an orthogonal part. The crucial issue is to establish the closedness of a properly defined space of stochastic integrals playing the role of $\widetilde{G}$ above.

We concentrate on the case of a continuous process $S$ but we do not assume that $S$ is $L_{2}$-bounded. We then may uniquely decompose $S$ into its local martingale and into its finite variation part

$$
S=S_{0}+M+A
$$

We have seen in section 4that under the condition of (NFLVR) the $\mathbb{R}^{d}$ valued measure $d A$ is absolutely continuous with respect to $d\langle M\rangle$ (taking values in the non-negative definite operators on $\mathbb{R}^{d}$ ) and we may find an $\mathbb{R}^{d}$-valued predictable process $\lambda=\left(\lambda_{t}\right)_{0 \leq t \leq T}$ such that

$$
d A_{t}=d\langle M\rangle_{t} \lambda_{t} \quad \mathbb{P} \text {-a.s. for } 0 \leq t \leq T .
$$

A formal application of the Girsanov-theorem indicates that the probability measure $\mathbb{Q}^{\text {min }}$ defined via the density process

$$
\text { and } \begin{aligned}
L_{t} & :=\mathcal{E}(-\lambda \cdot M)_{t}, \\
\frac{d \mathbb{Q}^{\text {min }}}{d \mathbb{P}}:=L_{T} & =\mathcal{E}(-\lambda \cdot M)_{T},
\end{aligned}
$$

where $\mathcal{E}$ denotes the Doléans-Dade exponential of a local martingale, is a candidate for an equivalent local martingale measure for the process $S$ (provided all the involved limiting procedures make good sense and $L_{T}$ really defines a 
density of an equivalent measure). Föllmer-Schweizer have called this measure the "minimal" martingale measure $\mathbb{Q}^{\text {min }}$ (if it exists).

Another natural choice from the set $\mathbb{M}^{e}(S)$ of equivalent local martingale measures for $S$ is $\mathbb{Q}^{\text {opt }}$, the "variance-optimal" measure, which minimizes the $L^{2}(\Omega, \mathcal{F}, \mathbb{P})$-norm among all elements of $\mathbb{M}^{e}(S)$ (again, provided it exists). In many cases, e.g., when $S$ has independent increments, $\mathbb{Q}^{\text {min }}$ and $\mathbb{Q}^{\text {opt }}$ coincide, but in general they are different.

We now turn again to the theme of closedness of a (properly defined) space of stochastic integrals.

To introduce the notion of predictable trading strategies appropriate in the present context, we call a predictable $\mathbb{R}^{d}$-valued process $L^{2}$-admissible if $H \cdot M$ as well as $H \cdot A$ make sense in $L^{2}$, i.e., if $H \cdot M$ is an $L^{2}(\Omega, \mathcal{F}, \mathbb{P})$-bounded martingale and $\mathbb{E}\left[\left(\int_{0}^{T}\left|H_{u} d A_{u}\right|\right)^{2}\right]<\infty$. The definition is chosen in such a way that we may define the stochastic integral $H \cdot S=H \cdot M+H \cdot A$ and that $(H \cdot S)_{T}$ is in $L^{2}(\Omega, \mathcal{F}, \mathbb{P})$. Denote again by $G$ the subspace of $L^{2}(\Omega, \mathcal{F}, \mathbb{P})$ formed by the functions $(H \cdot S)_{T}$, where $H$ runs through the $L^{2}$-admissible trading strategies.

To formulate the theorem we recall that a measure $\mathbb{Q} \sim \mathbb{P}$ with density process $L_{t}=\mathbb{E}\left[\frac{d \mathbb{Q}}{d \mathbb{P}} \mid \mathcal{F}_{t}\right]$, satisfies the reverse Hölder condition $R_{p}$, for $1<p \leq$ $\infty$, if there is a constant $C$ such that

$$
\mathbb{E}\left[\left(\frac{d \mathbb{Q}}{d \mathbb{P}}\right)^{p} \mid \mathcal{F}_{t}\right] \leq C \mathbb{E}\left[\frac{d \mathbb{Q}}{d \mathbb{P}} \mid \mathcal{F}_{t}\right]^{p}, \quad \text { for } 1 \leq t \leq T .
$$

The name stems from the fact that by Hölder's inequality the reverse inequality is always satisfied with $C=1$. The reverse Hölder condition is dual to the Muckenhaupt condition (see [DDM79]).

We now can formulate the central result of [DMSSS97]:

Theorem 6.1 Let $S=\left(S_{t}\right)_{0 \leq t \leq T}$ be a continuous semi-martingale and suppose that there exists an equivalent probability measure $\mathbb{Q}$ with $\frac{d \mathbb{Q}}{d \mathbb{P}} \in L^{2}(\Omega, \mathcal{F}, \mathbb{P})$ under which $S$ is a local martingale.

The space $G$ is closed in $L^{2}(\Omega, \mathcal{F}, \mathbb{P})$ if and only if there is an equivalent local martingale measure $\mathbb{Q}$ for $S$ which satisfies the reverse Hölder condition $R_{2}$. In this case the so-called "variance-optimal" measure $\mathbb{Q}^{\mathrm{opt}}$, which has minimal $L^{2}(\Omega, \mathcal{F}, \mathbb{P})$-norm among all equivalent local martingale measures, is welldefined and satisfies the reverse Hölder condition $R_{2}$.

There are some variants of the above theorem in terms of the process $\lambda \cdot M$. A necessary condition for theorem 6.1 to hold true (under the assumptions given there) is that the process $\lambda \cdot M$ is in BMO but this condition is not sufficient. In fact $\lambda \cdot M$ is in BMO is equivalent to the completeness of $G$ with 
respect to the stronger norm defined via the maximal function

$$
\|(H \cdot S)\|:=\left\|\sup _{t}(H \cdot M)_{t}\right\|_{L^{2}(\Omega, \mathcal{F}, \mathbb{P})} .
$$

On the other hand the condition that the minimal measure $\mathbb{Q}^{\text {min }}$ defined via $\frac{d \mathbb{Q}^{\text {min }}}{d \mathbb{P}}=\mathcal{E}(-\lambda \cdot M)$ exists and satisfies the reverse Hölder condition $R_{2}$ is sufficient but not necessary for the theorem to hold true. In fact, this stronger condition is equivalent to the fact that $G$ is closed and, in addition, that the (in general not orthonormal) projection $\pi$ from $L^{2}(\Omega, \mathcal{F}, \mathbb{P})$ onto $\widetilde{G}$ with $\operatorname{ker}(\pi)=M^{\perp}$ is well defined and continuous. Here $M^{\perp}$ denotes the subspace of $L^{2}$ orthogonal to the space $M$ generated by the constants and the stochastic integrals on the local martingale part $M$ of the process $S$. If $\pi$ is welldefined and bounded then $\pi$ induces the Föllmer-Schweizer decomposition as it splits a general contingent claim $f \in L^{2}(\Omega, \mathcal{F}, \mathbb{P})$ into the hedgeable part $\pi(f)$ which may be written as $\pi(f)=$ const $+\int_{0}^{T} H_{u} d S_{u}$ for an $L^{2}$-admissible trading strategy $H$ and the non-hedgeable part $f-\pi(f)$ which induces a martingale strongly orthogonal to the local martingale part $M$ of $S$.

We refer to [DMSSS97] for a detailed presentation of these topics for the above discussed closedness of $G$ in $L^{p}$ for $p=2$ and the case of $R^{d}$-valued continuous semi-martingales $S$. Extensions to the case $1<p<\infty$ as well as to the case of general $R^{d}$-valued semi-martingales were obtained in [GK98], [CKS97] and [CKS98]. 


\section{References}

[AS94] Ansel, J.P., Stricker, C. Couverture des actifs contingents et prix maximum. Ann. Inst. Henri Poincaré 30, 1994, pp. 303-315.

[B00] Bachelier, L. Théorie de la spéculation. Ann. Sci École Norm. Sup. 17 (1900), 21-86. [English translation in: The Random Character of Stock Market Prices, P.Cootner, ed. MIT Press, Cambridge (Mass.) 1964, pp. 17-78.

[B98] Björk, T. Arbitrage Theory in Continuous Time.Oxford University Press (1998).

[BS73] Black, F., Scholes, M. The pricing of options and corporate liabilities. J. Political Econom. 81, 1973, 637-654.

[Ch00] Cheridito, P. Arbitrage for fractional Brownian motion. ETH working paper (2000).

[CKS97] Choulli, T., Krawczyk, L., Stricker, C. (1997) On Fefferman and Burkholder-Davis-Gundy inequality for E-martingales. to appear in Prob. Theory and Related Fields.

[CKS98] Choulli, T., Krawczyk, L., Stricker, C. (1998) E-martingales and their applications in mathematical finance. Ann. Probability 26, pp. 853876.

[D92] Delbaen, F. (1992) Representing martingale measures when asset prices are continuous and bounded. Math. Finance 2, 107-130.

[DMW90] Dalang, R.C., Morton, A., Willinger, W. (1990) Equivalent martingale measures and no-arbitrage in stochastic securities market model. Stochastics Stochastics Rep. 29, 185-201.

[DMSSS97] Delbaen, F., Monat, P., Schachermayer, W., Schweizer,M., Stricker, C. (1997) Weighted norm inequalities and hedging in incomplete markets. Finance and Stochastics, Vol. 1, No. 3 (1997), pp. 181-227.

[DS94] Delbaen, F., Schachermayer, W. (1994) A General Version of the Fundamental Theorem of Asset Pricing. Math. Annalen 300, p. 463520.

[DS95] Delbaen, F., Schachermayer, W. The Existence of Absolutely Continuous Local Martingale Measures. Annals of Applied Probability, Vol. 5., No. 4, (1995), pp. 926-945. 
[DS95a] Delbaen, F., Schachermayer, W. An Inequality for the Predictable Projection of an Adapted Process. Sem. de Probabilits XXIX, Springer LNM 1613, (Eds. J. Azma, M. Emery, P.A. Meyer, M. Yor) (1995), pp. 17-24.

[DS97] Delbaen, F., Schachermayer, W. The Banach Space of Workable Contingent Claims in Arbitrage Theory. Annales de l' I.H.P., Vol. 33, No. 1 (1997), pp. 113-144.

[DS98] Delbaen, F., Schachermayer, W. The Fundamental Theorem of Asset Pricing for Unbounded Stochastic Processes. Mathematische Annalen 312 (1998), pp. 215-250.

[DS98a] Delbaen, F., Schachermayer, W. A simple Counterexample to Several Problems in the Theory of Asset Pricing. Mathematical Finance, vol 8, 1998, pp. 1-12.

[DM80] Dellacherie, C., Meyer, P.-A. (1980) Probabilités et potentiel. Théorie des martingales. Hermann, Paris.

[DDM79] Doléans-Dade, C., Meyer, P.A. inégalitis de normes avec poids. Sém. de Probabilité XIII, pp. 313-331, Lecture Notes 721, Springer 1979

[DH86] Duffie, D., Huang, C.-F. (1986) Multiperiod security markets with differential information; martingales and resolution times. J. Math. Econom. 15, 283-303.

[D93] Dupire B. Model Art. Risk, vol. 6, no. 9, Sept 1993

[E05] A. Einstein On the moment of small particles suspended in stationary liquid demanded by molecular-kinetic theory of heat, Ann. Phys. 17, 1905.

[E06] A. Einstein Zur Theorie der Brownschen Bewegung., Ann. Phys. 19, pp. 371-381, 1906.

[E56] A. Einstein Investigations on the Theory of the Brownian Movement. Dover, New York, 1956.

[EQ95] El Karoui, N., Quenez, M.C. (1995) Dynamic programming and pricing of contingent claims in an incomplete market. SIAM J. Control Optimiz. 33, 29-66.

[E80] M. Emery Compensation de processus á variation finie non localement intégrables. Sém. de Probabilité XIV, LNM vol. 784, pp. 5260,1980 
[FSch91] Föllmer, H., Schweizer, M. (1991) Hedging of contingent claims under incomplete information. In: Applied Stochastic Analysis, M.H.A.Davis and R.J.Elliott, eds. Gordon and Breach, London New York, pp. 389-414.

[FS86] Föllmer, H., Sondermann, D. (1986) Hedging of non-redundan contingent claims. In: Contributions to Mathematical Economics in Honor of Gérard Debreu, W.Hildenbrand and A.Mas-Colell, eds. North Holland, Amsterdam, pp. 205-223.

[GK98] Grandits, P., Krawczyk, L. Closedness of some spaces of stochastic integrals. Seminaire de Probabilites XXXII, 1998, pp. 73-85.

[HK79] Harrison, J.M., Kreps, D.M. (1979), Martingales and arbitrage in multiperiod securities markets. J. Econom. Theory 20, 381-408.

[HP81] Harrison, J.M., Pliska, S.R. (1981), Martingales and stochastic integrals in the theory of continuous trading. Stochastic Process. Appl. $11,215-260$.

[J92] Jacka, S.D. (1992) A martingale representation result and an application to incomplete financial markets. Math. Finance 2, 239-250.

[J79] Jacod, J. (1979) Calcul stochastique et problèmes de martingales. Lecture Notes in Math. 714. Springer, Berlin Heidelberg New York.

[KS98] Karatzas, I., Shreve, S. Methods of Mathematical Finance. Springer (1997).

[K33] Kolmogoroff, A.N. Grundbegriffe der Wahrscheinlichkeitsrechnung. Erg. Math 2, 1933, Springer

[K81] Kreps, D. (1981) Arbitrage and equilibrium in economies with infinitely many commodities. J. Math. Econom. 8, 15-35.

[LL96] Lamberton, D., Lapeyre, B. Introduction to Stochastic Calculus Applied to Finance. Chapman \& Hall (1996).

[LS95] Levental, S., Skorohod, A.S. A necessary and sufficient condition for absence of arbitrage with tame portfolios. Ann. Appl. Probab. 5, (1995), pp. 906-925.

[M66] Mandelbrot, B.B. Forecasts of Future Prces, Unbiased Markets and "Martingale" Models. Journal of Business, 39, pp. 242-255, 1966

[M80] J. Memin Espaces de semi-martingales et changement de probabilité. Zeitschrift für Wahrscheinlichkeitstheorie und verwandte Gebiete, vol.52, 1980, pp. 9-39. 
[M73] Merton, R.C. (1973), Theory of rational option pricing. Bell J. Econom. Manag. Sci. 4, 141-183.

[MR97] Musiela, M., Rutkowski, M. (1997) Martingale Methods in Financial Modelling. Applications of Mathematics. Springer (1997).

[P90] Protter, P. (1990) Stochastic Integration and Differential Equations. Springer, Berlin Heidelberg New York.

[RY91] Revuz, D., Yor, M. (1991), Continuous Martingales and Brownian Motion. Springer, Berlin Heidelberg New York.

[RW87] Rogers, L.C.G., Williams, D. (1987) Diffusions, Markov Processes and Martingales, Volume 2: Itô Calculus. Wiley, Chichester New York.

[R97] Rogers, L.C.G. (1997), Arbitrage from fractional Brownian motion. Math. Finance 7, pp. 95-105.

[S65] Samuelson, P.A. Proof that Properly Anticipated Prices Fluctuate Randomly. Industrial Management Review 6, pp. 42-49, 1965.

[S93] Schachermayer, W. A Counter-Example to several Problems in the Theory of Asset Pricing. Math. Finance 3, (1993), pp. 217-229.

[S94] Schachermayer, W. Martingale Measures for discrete time processes with infinite horizon. Math. Finance, Vol. 4, No. 1 (1994), pp. 25-55.

[S99] Shiryaev, A.N. Essentials of Stochastic Finance. Facts, Models, Theory. World Scientific (1999).

[Sm16] Smoluchowski, M. Drei Vorträge über Diffusion, Brownsche Bewegung und Koagulation von Kolloidteilchen. Phys.Z. 17, 1916, pp. 557585.

[S90] Stricker, C. (1990) Arbitrage et lois de martingale. Ann. Inst. H.Poincaré Probab. Statist. 26, 451-460.

[Yan80] Yan, J.A. Caracterisation d' une classe d'ensembles convexes de $L^{1}$ ou $H^{1}$. Seminaire de Probabilites XIV, Lect. Notes Mathematics, vol. 784, 1980, pp. 220-222. 